\title{
Metodología para la simulación acelerada del deterioro que por corrosión atmosférica se presenta en equipo electrónico
}

\author{
A. Ortiz-Prado, R. Schouwenaars y S.M. Cerrud-Sánchez \\ Departamento de Ingeniería Mecánica \\ Facultad de Ingeniería, UNAM \\ E-mail:cerrud@servidor.unam.mx,
}

(recibido: julio de 2001 ; aceptado: mayo de 2002)

\begin{abstract}
Resumen
Para la evaluación de la resistencia a la corrosión de sistemas y equipo electrónico que trabajarán bajo condiciones de deterioro generadas por el medio ambiente, se han aplicado una serie de ensayos, donde el más popular es el de cámara de niebla salina. Sin embargo, éste y otros que se han elaborado para tal efecto no tienen ninguna relación con las condiciones reales de servicio, por lo que es necesario un método de evaluación que permita simular de forma acelerada los fenómenos de deterioro por efectos ambientales. Este artículo pretende demostrar la necesidad de desarrollar una prueba, que en forma acelerada, reproduzca el daño que sufre el material por efecto de la atmósfera; el cual se orienta a la evaluación de equipo eléctrico y electrónico.
\end{abstract}

Descriptores: corrosión, equipo electrónico, deterioro acelerado.

\section{Abstract}

The corrosion resistance of systems and electronic parts which are designed to work in atmospheric conditions have been tested for decades; some of these methods were the Cyclic Humidity Test. Field Tests and Salt Spray (Fog) Testing, the latter was one of the most popular methods. However, the salt spray test and most of the other existing methods do not show strong relationships with the real conditions of service. For this reason it is necessary to develop appropriated methods and equipment for the accelerated simulation of real atmospheric corrosion phenomena. This article seeks to demonstrate the need to develop a test and the necessary equipment to reproduce the damage in electronic systems and equipment by atmospheric corrosion.

Keywords: corrosion, electronic equipment, accelerated damage.

\section{Introducción}

El equipo y partes electrónicas expuestos a la atmósfera sufren daños directamente por corrosión y por problemas derivados de la propia interacción de la humedad, gases contaminantes, cloruros y agentes catalíticos como el ozono y la luz ultravioleta. Estos deterioros dependen de la composición característica de la atmósfera (tipos de contaminantes y su concentración), variación de la temperatura, así como de la sensibilidad de los materiales y recubrimientos a las condiciones del ambiente.

Los principales contaminantes gaseosos, desde el punto de vista de corrosión atmosférica son el $\mathrm{O}_{3}$, 
$\mathrm{SO}_{2}, \mathrm{CO}_{2}, \mathrm{NO}_{x}$ y el $\mathrm{Cl}$ en varias formas. Éstos se encuentran en cantidades abundantes en regiones donde existe una alta densidad de población y concentración de la planta productiva. Además de los contaminantes mencionados, tienen un papel importante, el grado de humedad del ambiente y la variación de temperatura, ya que en regiones cercanas a las costas, los iones cloro se encuentran en grandes concentraciones, factor que facilita el proceso de corrosión.

Durante la guerra de Vietnam, el equipo electrónico norteamericano de comunicaciones y armamento, presentó diversas fallas, las cuales tuvieron su origen en fenómenos corrosivos, debidos a las condiciones ambientales extremas que caracterizan dicha región. Como consecuencia de estas observaciones, se inició el estudio sobre corrosión en equipo electrónico.

Guttenplan (1987), detectó la problemática de la corrosión en la industria electrónica, de acuerdo a las siguientes tendencias:

a) Mayor densidad de componentes con menor tamaño y espaciamiento, así como menores secciones metálicas.

b) Requerimientos de pistas con baja resistencia

c) Exposición del equipo electrónico a condiciones más severas.

De lo antes expuesto y de lo observado por otros autores, se concluye que pequeñas cantidades de contaminantes o de productos de corrosión pueden causar daños que conllevan a la falla del equipo. Niveles bajos de humedad combinados con la presencia de contaminantes corrosivos pueden causar problemas en circuitos impresos, circuitos integrados, conectores eléctricos, potenciómetros y otros componentes electrónicos.

La corrosión en equipo electrónico puede ocurrir durante la manufactura, almacenaje, transportación o durante el servicio. Los principales agentes corrosivos identificados son la humedad, los cloruros, fluoruros, $\mathrm{H}_{2} \mathrm{~S}$, dióxido de azufre, compuestos de nitrógeno como el amoniaco y las partículas suspendidas. El ambiente al que están expuestos los circuitos electrónicos proviene de dos fuentes: los materiales con los que está construido el equipo y la atmósfera en sí misma.

Gouda et al. (1989), estudió casos de corrosión en componentes de hardware mediante análisis por microscopía electrónica y EDS (Energy Dispersive Spectroscopy), determinando concentraciones que de gases contaminantes 10 veces menores al límite permitido para el hombre, pueden dañar el equipo electrónico. Los laboratorios de aeronáutica de la fuerza aérea norteamericana (White et al., 1987) han estudiado varios casos de falla por corrosión en sistemas de este tipo. Con estos estudios se concluyó que el $80 \%$ de las fallas eléctricas y electrónicas se deben a defectos en materiales y procesos de manufactura, y el $20 \%$ se deben específicamente a problemas de corrosión.

El fenómeno de la corrosión atmosférica se inicia a partir de la formación de una capa acuosa en la superficie del material, la cual proviene de la humedad del ambiente y está determinada por precipitaciones pluviales, niebla o cualquier otro factor climatológico. Como puede verse en Rodríguez et al. (1999), una vez que se presenta dicha capa y que actúa a modo de solvente para ciertos contaminantes se da lugar a la acidificación de la solución, con lo que se manifiesta una aceleración en las reacciones electroquímicas, teniendo como resultado la degradación del material.

Factores que determinan la corrosividad atmosférica

La corrosión atmosférica es uno de los fenómenos de deterioro más estudiado, y a la fecha, todavía se tienen dudas en cuanto a su modelado y caracterización mediante ensayos en el laboratorio; sin embargo, ha sido definida como la degradación que sufren los materiales, que están en contacto con el aire, y por ende, con los constituyentes y contaminantes del mismo.

Los factores más importantes son:

a) Variación de temperatura. 
b) Ciclos de humedad.

c) Contaminantes gaseosos presentes en el aire.

- Composición y concentración.

d) Partículas contaminantes.

- Composición, tamaño y concentración.

Para evaluar el comportamiento de los materiales durante su exposición en la atmósfera, se estructuran diversos tipos de ensayos, éstos se clasifican en:

1. Las pruebas de campo: son aquellas en las que el testigo es expuesto a las variables existentes en el ambiente sin que éstas puedan ser manipuladas.

2. Las pruebas de laboratorio: en las que ciertas variables pueden ser controladas. Entre este tipo de pruebas se encuentran las siguientes:
a) Cámara de niebla salina
b) Cámara de niebla salina + ácido acético
c) Cámara de niebla salina $+\mathrm{SO}_{2}$
d) Cámara acelerada de niebla salina $+\mathrm{Cu}^{+2}$
e) Cámara de ciclos de humedad
f) Cámara de gases

Para la mejor comprensión de cómo afecta el medio ambiente, se han agrupado las condiciones climatológicas y los contaminantes en diferentes tipos de ambiente. Los principales son:

Ambiente industrial urbano: Se caracteriza por tener todo tipo de contaminación por azufre, principalmente en forma de dióxido de azufre, el cual proviene en su mayoría de la combustión de combustibles fósiles. Adicionalmente, a este contaminante se encuentra el $\mathrm{NO}_{\mathrm{x}}$.

Ambiente marino: Posee altas concentraciones de iones cloro. La concentración de $\mathrm{NaCl}$ en este ambiente se mantiene superior a los $15 \mathrm{mg} / \mathrm{m}^{2}$ día, no importando el nivel de $\mathrm{SO}_{2}$ que se presente. La corrosividad se puede ver incrementada por la existencia de industria en la zona, así como por la temperatura y la dirección de los vientos dominantes.
Ambiente rural: No tiene las concentraciones de contaminantes presentadas en los otros tipos de ambientes, ya que en estas áreas no existe una alta densidad de población, ni de industria; sin embargo, el empleo de fertilizantes y la descomposición de materia orgánica activa otros mecanismos de corrosión debidos a la presencia de iones amonia, nitratos, etc.

Existen subdivisiones de estos tipos de ambientes, como tropical, subtropical, ártico, húmedo, seco, templado, entre otros.

\section{Efecto de la corrosión en el funcionamiento del equipo electrónico}

Los fabricantes de equipo electrónico, en particular telefónico, han observado que los aparatos presentan fallas en el servicio por efecto del medio ambiente; razón por la que se han utilizado una serie de ensayos mediante los que se pretende evaluar este efecto. En algunos casos, las pruebas no corresponden de ninguna forma con las condiciones de servicio (cámara de niebla salina) y en otras, es prácticamente imposible correlacionar el tiempo de ensayo con el de servicio. Las condiciones ambientales, aún en interiores, no se pueden generalizar para cualquier país o región, por lo que es necesario desarrollar estándares de prueba que se adecuen a las condiciones más comunes y agresivas.

Como muestra de la variabilidad de las condiciones ambientales se puede mencionar el caso de México, cuyo clima es muy diverso debido a la topografía y altitud, pasando desde tropical lluvioso a templado y hasta desértico. Las condiciones ambientales varían durante el año. Las temperaturas mínimas y máximas absolutas en México son de $-20^{\circ} \mathrm{C}$ y $50^{\circ} \mathrm{C}$ respectivamente, mientras que la humedad relativa varía entre los 30\% y $100 \%$. Por otra parte, la diversidad de los contaminantes presentes y su concentración, por ejemplo en la zona metropolitana de la Ciudad de México, da como resultado que equipos del mismo tipo puedan estar instalados en regiones de distinta corrosividad. 
El problema radica en identificar el efecto que el medio ambiente tiene en el desempeño del equipo electrónico, con el fin de conocer las limitaciones en la vida útil del aparato, lo cual se puede lograr al efectuar pruebas de simulación que reproduzcan los efectos de las condiciones atmosféricas y de calidad del aire.

\section{Variables principales del daño por corrosión atmosférica. Su efecto y procedencia}

Como se ha mencionado, la corrosión atmosférica depende de la humedad del ambiente, las condiciones extremas de temperatura y los contaminantes que se encuentran dispersos en el aire. Dada la variabilidad de estos factores, es prácticamente imposible realizar un modelado matemático que permita determinar con precisión el comportamiento del material; por esta razón se torna inviable correlacionar el tiempo de ensayo con el tiempo de exposición real. Por otra parte, la variación de condiciones ambientales en interiores es menos dramática, aun cuando pueda existir una mayor concentración de ciertos contaminantes, efecto de las propias actividades domésticas.

Para determinar la corrosividad de una atmósfera es necesario relacionar entre sí todos los factores que la afectan, en especial aquellos que han demostrado tener particular influencia en el proceso de corrosión atmosférica:

a) Tiempo de humectación de la superficie metálica.

b) Cantidad de contaminantes que pueden depositarse en la superficie metálica.
De acuerdo a la norma ISO 9223:1992a, se consideran como elementos clave de la corrosión:

a) Tiempo de humectación

b) Los niveles de $\mathrm{SO}_{2}$ y $\mathrm{NaCl}$

Dentro de los tipos de contaminantes que pueden ejercer un efecto agresivo, se encuentran el $\mathrm{No}_{x}$, $\mathrm{SO}_{\mathrm{x}}$ en zonas industriales, $\mathrm{CaCl}_{2}$ y $\mathrm{MgCl}_{2}, \mathrm{NaCl}$ en las regiones marítimas y otros específicos de un determinado microclima como el cloro y ácidos orgánicos, entre otros.

Tiempo de humectación. La determinación del tiempo de humectación de una superficie que se corroe $(\tau)$, se efectúa considerando aquellos períodos en que la humedad relativa es mayor de $80 \%$ y la temperatura superior a $0^{\circ} \mathrm{C}$; de acuerdo con esto, el tiempo de humectación se puede obtener a partir de datos climáticos.

Nivel de $\mathrm{SO}_{2}$. La velocidad de deposición de $\mathrm{SO}_{2}$ se determina mediante el método de los platos de sulfatación, especificada en la norma ISO 9225: 1992.

Procesos por cloruros. La clasificación de la contaminación por pulverizaciones o aerosoles salinos, representada por el nivel de $\mathrm{NaCl}$, se relaciona con atmósferas exteriores en ambientes marinos. Para la determinación de este dato se puede utilizar el método de la candela húmeda o la norma DIN 50907-1952

En la tabla l se presentan las fuentes más comunes de contaminantes en la corrosión atmosférica.

Tabla 1. Algunas fuentes comunes de constituyentes reactivos del medio ambiente de acuerdo con la norma ANSI/ISA-S7 1.04-1985

Constituyente

\begin{tabular}{|c|c|c|}
\hline & Constituyente & Fuentes comunes \\
\hline $\mathrm{H}_{2} \mathrm{~S}$ & Ácido sulfhídrico & $\begin{array}{l}\text { Emisiones geotérmicas, actividades microbiológicas, procesamiento de combustibles fósiles, pulpa de } \\
\text { madera, tratamiento de aguas de desecho, combustión de combustibles fósiles, fusión de minerales, } \\
\text { emisiones de autos, manufactura de ácido sulfúrico }\end{array}$ \\
\hline $\mathrm{SO}_{\mathrm{x}}$ & Óxidos de azufre & $\begin{array}{l}\text { Combustión de combustibles fósiles, emisiones de autos, fusión de minerales, manufactura de ácido } \\
\text { sulfúrico, humo de tabaco }\end{array}$ \\
\hline $\mathrm{HF}$ & Ácido fluorhídrico & $\begin{array}{l}\text { Manufactura de: fertilizantes, aluminio, cerámicos, aceros, dispositivos electrónicos, combustibles } \\
\text { fósiles }\end{array}$ \\
\hline
\end{tabular}


DOI: http://dx.doi.org/10.22201/fi.25940732e.2002.03n4.015

A. Ortiz-Prado, R. Schouwenaars y S.M. Cerrud-Sánchez

Tabla 1. Algunas fuentes comunes de constituyentes reactivos del medio ambiente de acuerdo con la norma ANSI/ISA-S71.04-1985 (continuación)

\begin{tabular}{|c|c|c|}
\hline $\mathrm{NO}_{x}$ & Óxidos de nitrógeno & Microbios, emisiones de automóviles, combustión de combustibles fósiles, industria química \\
\hline $\mathrm{N}_{2}$ & $\begin{array}{l}\text { Nitrógeno orgánico } \\
\text { activado }\end{array}$ & Desperdicio de animales, combustión de vegetales, aguas negras, pulpa de madera \\
\hline $\mathrm{NH}_{3}$ & Amoniaco & $\begin{array}{l}\text { Microbios, aguas negras, manufactura de fertilizantes, vapor geotérmico, equipo de refrigeración, } \\
\text { productos de limpieza, máquinas heliográficas }\end{array}$ \\
\hline C & Carbono (carbón) & Combustión incompleta (constituyente de aerosoles), fundición \\
\hline $\mathrm{CO}$ & $\begin{array}{l}\text { Monóxido de } \\
\text { carbono }\end{array}$ & Combustión, emisiones de automóviles, microbios, árboles y pulpa de madera \\
\hline $\mathrm{Cl}_{2}, \mathrm{ClO}_{2}$ & $\begin{array}{l}\text { Cloro } \\
\text { Dióxido de cloro }\end{array}$ & $\begin{array}{l}\text { Manufactura de cloro, manufactura de aluminio, molinos de papel, descomposición de desechos, } \\
\text { productos de limpieza }\end{array}$ \\
\hline $\mathrm{HCl}$ & Ácido clorhídrico & Emisiones de automóviles, combustión, combustión de polímeros \\
\hline$\underset{\mathrm{HI}}{\mathrm{HBr}}$ & $\begin{array}{l}\text { Componentes } \\
\text { Halogenados }\end{array}$ & Emisiones de automóviles \\
\hline $\mathrm{Cl}^{-}$ & lones cloruro & Contenido de aerosoles, procesos oceánicos, procesamiento de minerales \\
\hline $\mathrm{O}_{3}$ & Ozono & $\begin{array}{l}\text { Procesos atmosféricos fotoguímicos que involucran óxidos de nitrógeno e hidrocarburos oxigenados, } \\
\text { emisiones de automóviles, filtros electrostáticos, etc. }\end{array}$ \\
\hline \multirow[t]{2}{*}{$\mathrm{C}_{n} \mathrm{H}_{\mathrm{n}}$} & Hidrocarburos & $\begin{array}{l}\text { Emisiones de automóviles, procesamiento de combustibles fósiles, humo de tabaco, tratamiento de } \\
\text { agua, microbios, fuentes naturales, molinos de papel }\end{array}$ \\
\hline & Polvo inorgánico & Cristal de roca, combustión, fuentes industriales diversas \\
\hline $\begin{array}{l}\mathrm{HCHO} \\
\mathrm{HCOOH}\end{array}$ & $\begin{array}{l}\text { Formaldehído } \\
\text { Acido fórmico }\end{array}$ & Adhesivos, humo de tabaco, combustión de biomasa, plásticos \\
\hline
\end{tabular}

\section{Corrosión atmosférica en interiores}

El conocimiento sobre corrosión en interiores es mucho menor comparado con la corrosión en exteriores. El estudio de la corrosión en interiores comenzó aproximadamente hace dos décadas y está dirigido principalmente al efecto de la corrosión en materiales electrónicos. Rice et al. (1980), estudiaron la corrosión en interiores de cobre, plata, níquel, cobalto y hierro en diferentes localidades de Estados Unidos. Ahí encontraron que, en general, la velocidad de corrosión es mucho menor en interiores, excepto en el caso de la plata para la cual es igual.

Se considera que existen diferencias importantes entre las condiciones en interiores y exteriores. La capa de electrolito formada en exteriores depende de las variaciones estacionales y diarias de humedad de la precipitación, rocío, nieve o niebla. En interiores, la capa formada depende de condiciones con menor variación del nivel de humedad, esto quiere decir, que en interiores los ciclos humedad-seco son menos notorios y drásticos, mientras que en las construcciones con poca protección del exterior es posible que las condiciones climáticas en el interior sean muy semejantes a las del exterior, sobre todo para equipo telefónico de servicio público en zonas pobres, por un ambiente tropical y semitropical.

La diferencia en la concentración de contaminantes en interiores y exteriores se debe principalmente a la concentración de gases y partículas en interiores y a la disminución de las variaciones atmosféricas exteriores por medio de sistemas de ventilación. Como se puede observar en la tabla 2, la concentración general de contaminantes en interiores es menor que en exteriores a excepción del amoniaco $\left(\mathrm{NH}_{3}\right)$ y el formaldehído (HCHO) (Lázaro, 1996). Esta diferencia particular se debe a la actividad antropogénica en interiores, ya que la mayor parte de los contaminantes encontrados en interiores provienen del exterior, aunque algunos se producen dentro como productos de calefacción, partículas sólidas, contaminación de estufas y calentadores de gas, nieblas de aceites, grasa de cocina o cigarros, esto según Lázaro (1996).

La tabla 2 sólo cumple funciones de comparación, ya que las concentraciones de los contaminantes varían según el ambiente. 
DOI: http://dx.doi.org/10.22201/fi.25940732e.2002.03n4.015

Tabla 2. Concentración general de contaminantes en interiores y exteriores

\begin{tabular}{cccc}
\hline & \multicolumn{2}{c}{ Compuesto } & \multicolumn{2}{c}{ Concentración (ppb) } \\
\cline { 3 - 4 } & Ozono & Exteriores & Interiores \\
\hline $\mathrm{O}_{3}$ & Agua oxigenada & $4-42$ & $3-30$ \\
$\mathrm{H}_{2} \mathrm{O}_{2}$ & Dióxido de azufre & $10-30$ & 5 \\
$\mathrm{SO}_{2}$ & Ácido sulfhídrico & $1-65$ & $0.3-14$ \\
$\mathrm{H}_{2} \mathrm{~S}$ & Dióxido de nitrógeno & $0.7-24$ & $0.1-0.7$ \\
$\mathrm{NO}_{2}$ & Ácido nítrico & $9-78$ & $1-29$ \\
$\mathrm{HNO}_{3}$ & Amoniaco & $1-10$ & 3 \\
$\mathrm{NH}_{3}$ & Ácido clorhídrico & $7-16$ & $13-259$ \\
$\mathrm{HCl}$ & Cloro & $0.18-3$ & $0.05-0.18$ \\
$\mathrm{Cl}_{2}$ & Formaldehído & $0.005-0.08$ & $0.001-0.005$ \\
$\mathrm{HCHO}$ & $-\ldots$ - & $4-15$ & 10 \\
$\mathrm{HCOOH}$ & $4-20$ & 20 \\
\hline
\end{tabular}

\section{Desarrollo de pruebas de corrosión para la industria electrónica}

Las pruebas aceleradas para simular el efecto de la corrosión tienen como objetivo determinar las velocidades de penetración del daño, para así proporcionar información que permita encontrar el tiempo de vida real de un producto, además de evaluar la problemática que las fallas por corrosión generan en el equipo. Con el trabajo de Campbell y Thomas (1930), se dio inicio al desarrollo de pruebas ambientales en equipo eléctrico y electrónico. A lo largo de 60 años se han desarrollado una gran variedad de métodos de prueba que, generalmente, incluyen uno o varios de los siguientes contaminantes: $\mathrm{SO}_{2}, \mathrm{O}_{3}, \mathrm{NO}_{x}, \mathrm{HCl}, \mathrm{NH}_{3}$ y $\mathrm{H}_{2} \mathrm{~S}$; también se han considerado las variables de humedad y temperatura, las cuales pueden permanecer constantes durante el ensayo o variar en forma cíclica, de esta manera es posible simular diferentes condiciones de trabajo.

En un principio se emplearon pruebas con altas concentraciones de un solo gas como dióxido de azufre ó $\mathrm{H}_{2} \mathrm{~S}$, desarrollándose también pruebas con mezclas de gases combinados con humedad. Algunas pruebas estandarizadas que utilizan un solo gas son las siguientes:

IEC Test $\mathrm{K}_{\mathrm{c}}, \mathrm{SO}_{2} \cdot{ }^{7}$

IEC Test $\mathrm{K}_{\mathrm{c}}, \mathrm{H}_{2} \mathrm{~S} .{ }^{8}$
JEIDA-32 sobre $\mathrm{SO}_{2} \cdot{ }^{9}$

JEIDA-25 sobre $\mathrm{H}_{2} \mathrm{~S}^{10}$

BS 2011 , Parte 2.1.11

DIN 40046 Partes 36 y 37.12

Otras pruebas de corrosión estandarizadas emitidas por la ASTM ${ }^{1}$ utilizadas para pruebas en sistemas electrónicos son:

\begin{tabular}{cl}
\hline Prueba & \multicolumn{1}{c}{ Descripción } \\
\hline B117 & Prueba de Niebla Salina \\
B287 & Prueba de Niebla Salina con Ácido Acético \\
B368 & Cooper-Accelerated Acetic Acid Salt Spray Fog Test \\
G87-84 & Standard Practice for Conducting Moist $\mathrm{SO}_{2}$ Test \\
\hline \hline
\end{tabular}

Las pruebas anteriores no presentan una correlación con el servicio real, ya que son generalmente sobre-simplificaciones del ambiente con condiciones de exposición severas, su empleo se limita al análisis comparativo del comportamiento de materiales y recubrimientos.

En 1986, Zakipour y Leygraf evaluaron diferentes pruebas de laboratorio que simulan la corrosión en interiores para materiales de contactos eléctricos, encontraron que se obtienen resultados cercanos a los esperados con mezclas de gases humectados con $\mathrm{SO}_{2}, \mathrm{NO}_{2}, \mathrm{NH}_{3}$ y $\mathrm{H}_{2} \mathrm{~S}$.

American Society for Testing Materials 
En 1997 Tidblad y Leygraf estudiaron el efecto de $\mathrm{SO}_{2}$, $\mathrm{NO}_{2}$ y ozono en ensayos para níquel, evaluando que el $\mathrm{NO}_{2}$ y el ozono promueven la corrosión al estar mezclados con $\mathrm{SO}_{2}$. Mientras que Oesch y Faller (1997), realizaron pruebas con ozono, $\mathrm{SO}_{2}, \mathrm{NO}$ y $\mathrm{NO}_{2}$, concluyendo que éste, además de ser un acelerador de la corrosión junto con otros contaminantes, es un fuerte agente corrosivo por sí solo.

\section{Resultados del estudio de daño por corrosión en aparatos telefónicos en servicio}

Para ratificar los datos obtenidos cuando se analizó el deterioro y daños sufridos en equipo electrónico instalado principalmente en el área metropolitana y en el estado de Morelos se tienen los resultados en la tabla 3; con ello, se puede concluir que aunque existan pares galvánicos, éstos no son críticos. En el caso de las soldaduras, existe evidencia de contaminación, pero en general, no se encontró ningún problema debido a este fenómeno; sin embargo, en las piezas de níquel, latón o con recubrimientos de este elemento, se encontraron depósitos que muestran daño por iones cloro, mientras que en los elementos recubiertos con plata, se presentan ataques por iones sulfato, teniéndose serios incovenientes por corrosión galvánica en presencia de oro.

\section{Condiciones que deberá cumplir un ensayo para la simulación acelerada de daño por corrosión atmosférica}

El ensayo deberá cumplir con lo siguiente:

\section{Continuidad}

2. Facilidad de implementación

3. Reproducir fenómenos que se presentan normalmente

4. Bajo costo
5. Rapidez

Características generales del ensayo propuesto:

1. Cámara ambiental.

2. Se tendrá la presencia de los gases contaminantes más comunes y que mayor efecto tienen.

3. Humedad relativa superior al $75 \%$.

4. Presencia de ozono como agente que promueve el fenómeno.

5. Los a paratos o tarjetas electrónicas a ser evaluadas podrán estar tanto en posición vertical como horizontal.

6. La temperatura variará cíclicamente para permitir condiciones de evaporación y condensación.

Con base en las observaciones realizadas y en los resultados de Zakipour y Leygraf (1986) y Guttenplan (1987), se propone que la prueba presente las siguientes peculiaridades:

1. Duración de la prueba: 200 ciclos.

2. Humedad relativa 85 a $100 \%$.

3. Gases contaminantes: $\mathrm{O}_{3}, \mathrm{SO}_{2}, \mathrm{NO}_{x}$, existiendo la posibilidad del $\mathrm{CO}_{2}$.

4. Concentración de gases contaminantes: Del orden de 100 veces la máxima para una atmósfera industrial.

5. Duración del ciclo de temperatura: 45 minutos.

6. Tipo de ciclo de temperatura: Onda trapezoidal, 0.75 del tiempo de ciclo a alta temperatura 0.25 del tiempo del ciclo a baja temperatura.

7. La temperatura máxima en grados centígrados será de 45 y la mínima de 5 .

8. La cámara dispondrá de ventiladores interiores de baja velocidad que garanticen la mezcla de los gases.

Tabla 3. Resultados obtenidos por medio de espectroscopía de rayos X con dispersión de energía en el microscopio electrónico de barrido.

\begin{tabular}{|c|c|c|c|c|c|c|}
\hline \multicolumn{7}{|l|}{ Productos de corrosión } \\
\hline Material & Latón & Plata & Níquel & Aluminio & Estaño & Lámina galvanizada \\
\hline Centro de la República & Cloruros & Sulfuros & Sulfuros & Ninguno & Ninguno & Ninguno \\
\hline Costa del Golfo & Cloruros & Sulfuros & & Ninguno & Óxidos & Óxidos / Nitratos \\
\hline
\end{tabular}


9. La cámara contará con deflectores que eviten la incidencia directa de la mezcla sobre las muestras.

10. La cámara contará con sistema de generación y control de atmósfera.

11. La cámara contará con un sistema de control de temperatura y humedad.

12. Los materiales de la cámara garantizarán que no exista deteriorio en éstos por efecto del medio empleado.

\section{Diseño conceptual del sistema}

De acuerdo con las características del ensayo, se determinó que para garantizar los ciclos de calentamiento y enfriamiento, lo más conveniente sería disponer de una bomba de calor (BC), descartándose el empleo de un ciclo de refrigeración convencional en conjunto con una resistencia para evitar problemas de corrosión que se pudieran suscitar en esta última, igualmente, se ha de garantizar el control del sistema mediante el empleo de la válvula de cuatro vías características de la $\mathrm{BC}$. El único problema lo representa la capacidad de los equipos comerciales, ya que el menor es de $18000 \mathrm{BTU} / \mathrm{hr}$, la cual supera por mucho los requerimientos dados por el volumen del aire a manejar $\left(1 \mathrm{~m}^{3}\right)$, y de la rapidez de calentamiento y enfriamiento( $10 \mathrm{~min}$ ), este aparente sobredimensionamiento del equipo permitirá que no se presente sobrecalentamiento en el fluido de trabajo de la bomba de calor. En cuanto a la presencia de humedad, se consideró más conveniente el empleo de un humidificador comercial, lo mismo para la generación del ozono. En lo referente a los gases contaminantes, se propone el empleo de gases industriales, requiriéndose sólo de electroválvulas para el suministro de los contaminante a la cámara; a partir de lo anterior, se propuso un sistema como el que se indica en la figura 1.

Para el diseño a detalle, fue necesario determinar primero la bomba de calor a utilizar, para que a partir de las medidas de la manejadora de aire correspondiente se definieran las de la estructura; por otro lado las de la cámara fueron precisadas con base al tamaño de los aparatos a manejar y al número de éstos que serán probados al mismo tiempo.

Con la finalidad de limitar el flujo de calor al exterior, el sistema se diseñó con una doble pared, donde se determinó que la cámara interior fuera construida en fibra de vidrio, esto para evitar problemas por corrosión, mientras que la exterior se determinó fuera de chapa de aluminio anodizado con el fin de garantizar la durabilidad y resistencia del sistema, para limitar los flujos de calor se propuso el empleo de espuma de poliestireno entre ambas paredes, la estructura de soporte del sistema se diseñó con base en perfilería de aluminio extruído (Figuras 2 y 3 ). En cuanto al sistema de monitoreo y control, éste se realiza a través de 3 sensores de temperatura y 2 de humedad, distribuidos en el interior de la cámara, los datos en el caso de humedad permiten el análisis del proceso, mientras que la señal de temperatura en conjunto con la de un temporizador permiten al programa el control de las funciones de la manejadora de aire y bomba de calor.

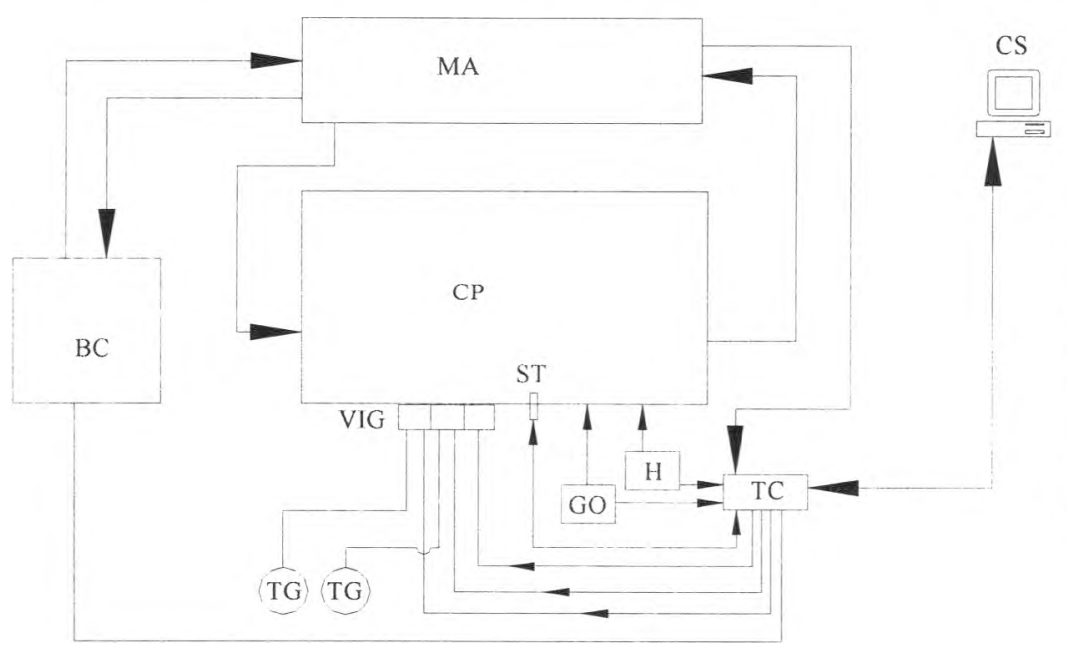

MA- Manejadora de Aire.

BC- Bomba de Calor.

CP-Cámara de Pruebas.

ST- Sensores de Temperatura.

VIG- Válvulas de Ingresos de

Gases.

TG- Tanque de Gas.

GO- Generador de Ozono.

H-Humidificador.

TC- Tarjetas de Control.

CS- Control del Sistema.

Figura 1. Esquema del sistema de pruebas aceleradas de corrosión 

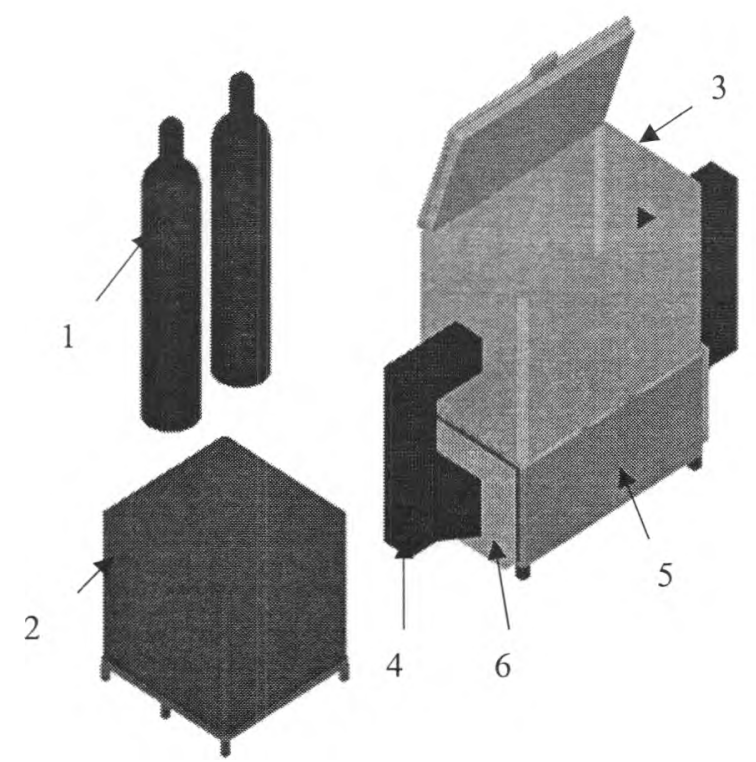

1.- Gases

2.- Bomba de calor

3.- Cámara

4.- Ducto de recirculación de aire

5.- Mesa de soporte de la cámara

6.- Manejadora de aire

Figura 2. Disposición del equipo de pruebas aceleradas de corrosión
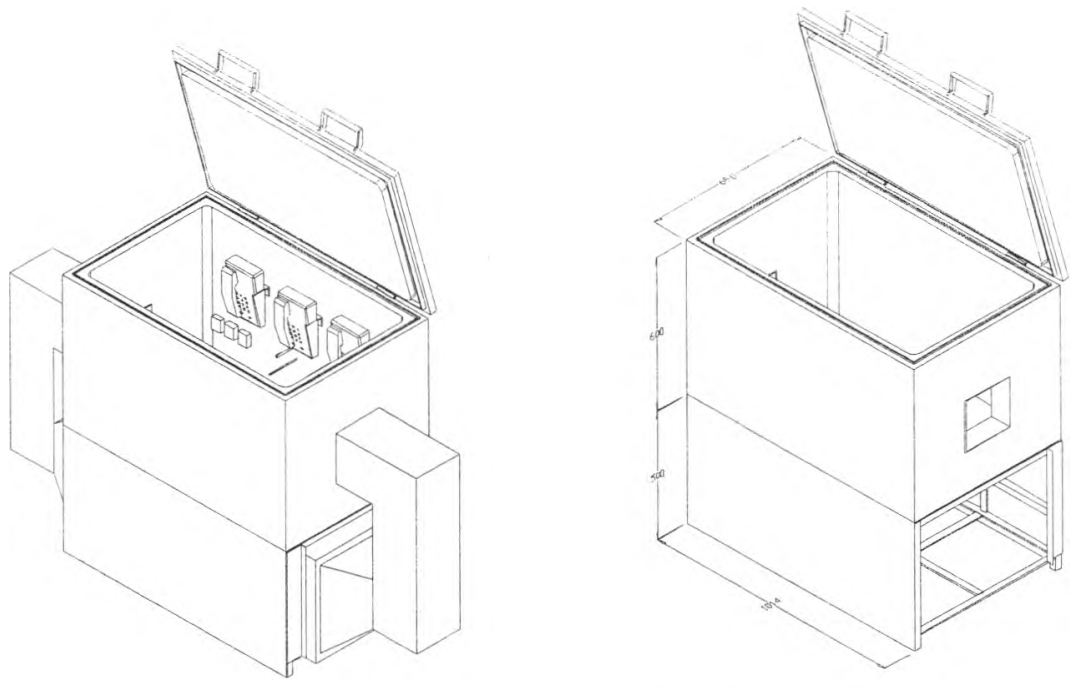

Figura 3. Cámara de pruebas aceleradas de corrosión

\section{Evaluación de la operación del sistema}

Para esta fase se colocaron dentro de la cámara tres tarjetas electrónicas de teléfono, una de ellas en posición vertical y las otras de manera horizontal, así como un teléfono nuevo. La prueba se realizó bajo las condiciones que se indican en la siguiente tabla:

\section{Condiciones de ciclo}

1. Calentamiento hasta $45^{\circ} \mathrm{C}$

2. Mantener temperatura alta durante $3 \mathrm{~min}$

3. Enfriamiento hasta $5^{\circ} \mathrm{C}$

4. Mantener temperatura baja durante $3 \mathrm{~min}$

Tolerancia del controlador: $\pm 1^{\circ} \mathrm{C}$

Operación de humidificador: 1000 [s] por ciclo

Operación del generador de ozono: 60 [s] por ciclo

Operación de la válvula: 1:3 [s] por prueba

Operación de la válvula: 2:3 [s] por prueba

Operación de la válvula: 3:0 [s] por ciclo 


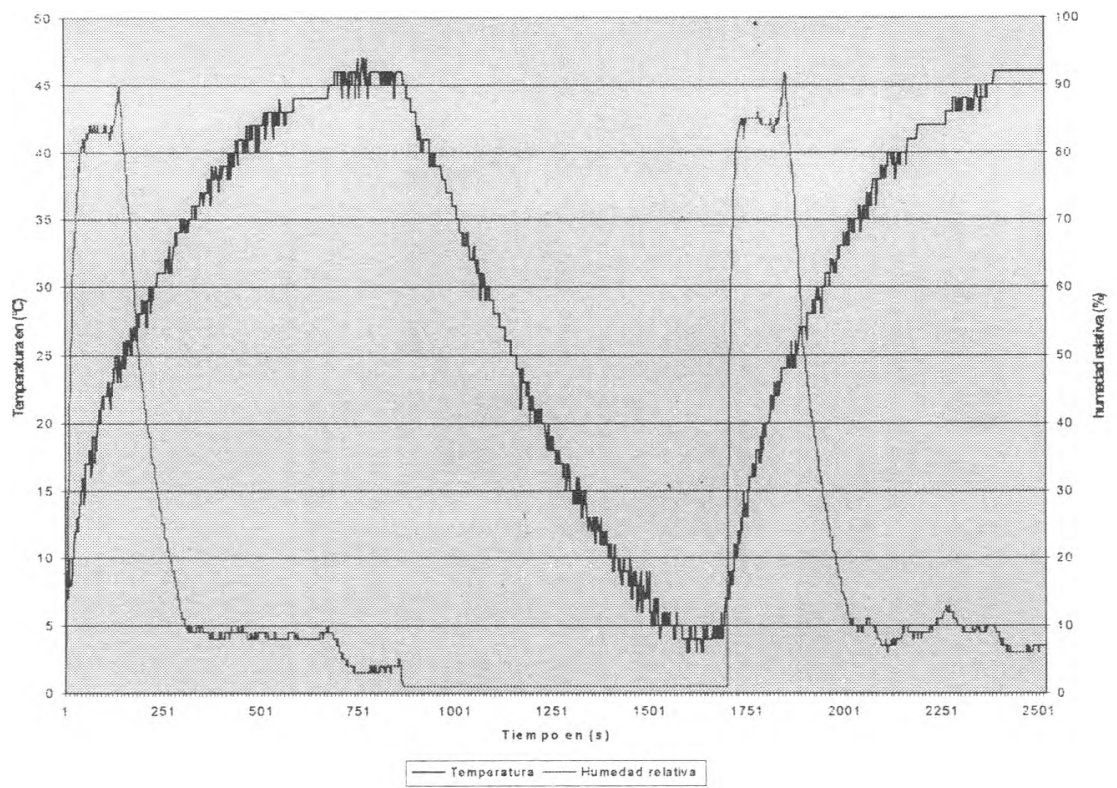

Figura 4. Ciclo de temperatura y humedad

En la figura 4 se muestra un ciclo de temperatura y humedad aplicado durante la prueba.

En la figura 5 se muestra una fotografía del daño en algunos componentes de las tarjetas electrónicas, percibiéndose la severidad de éste.

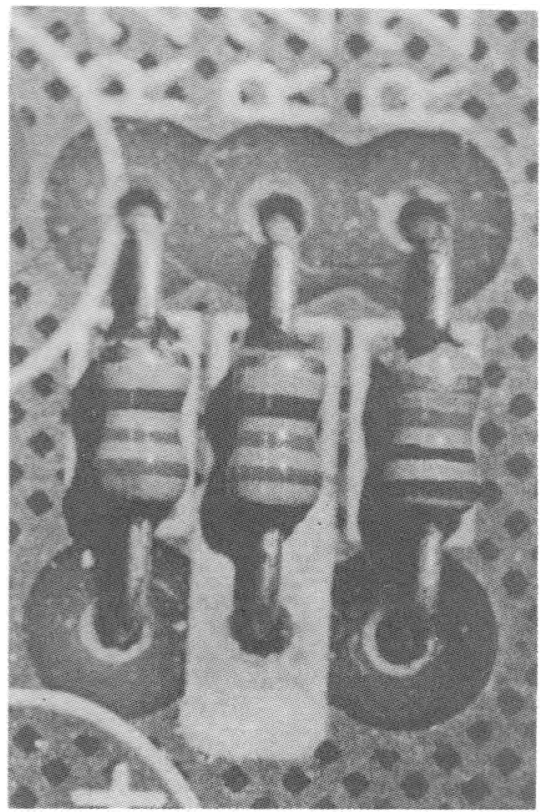

Figura 5. Daño en componentes electrónicos

\section{Conclusiones}

Con base en la necesidad de un equipo que simule el deterioro que el medio ambiente provoca en sistemas electrónicos, y considerando que en la actualidad no existe una prueba que combine el efecto de los agentes más comunes y agresivos, se ha planteado un nuevo ensayo de corrosión. Asimismo, se ha diseñado un equipo que puede realizar la prueba propuesta de manera controlada y repetitiva, en donde a la fecha, el sistema ya fue construido (Figura 6) y se encuentra en operación, solamente falta comparar el daño producido y observado en las tarjetas electrónicas en sistemas con varios años de servicio. Lo anterior permitirá la validación y ajuste de la prueba con la finalidad de que sirva como base para la respectiva norma nacional.

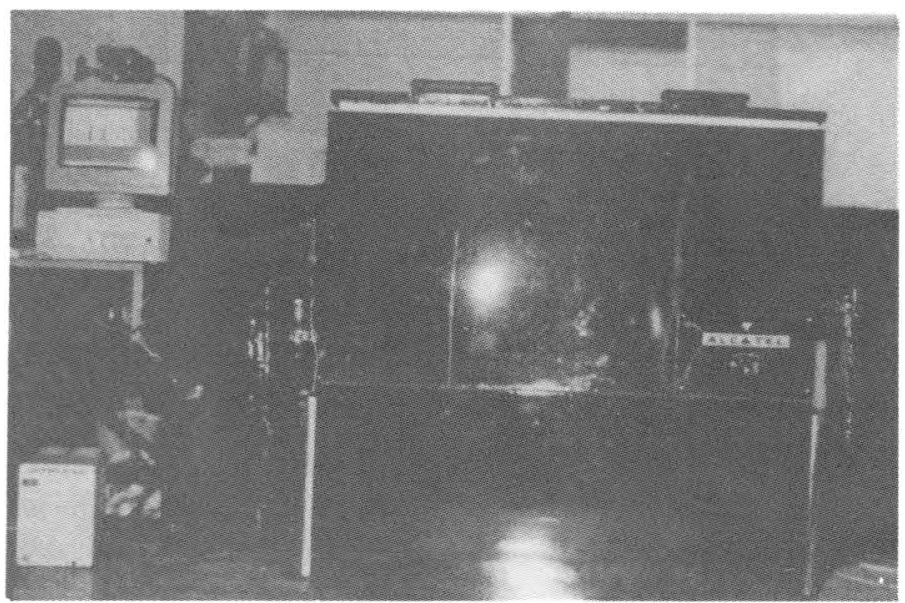

Figura 6. Disposición física de la cámara de pruebas 


\section{DOI: http://dx.doi.org/10.22201/fi.25940732e.2002.03n4.015}

\section{Referencias}

British Standard BS 2011. (1971). Parte 2.1, BSI, Londres.

German Standard DIN 40046. (1979). Partes 36 y 37 , Beunth, Berlin.

Gouda V.K., Carew J.A. y Riad W.D. (1989). Investigation of Computer Hardware Failure Due to Corrosion. British Corrosion I, Vol. 24, 192.

Guttenplan J.D. (1987). Corrosion in the Electronics Industry. Metals Hand6ook, ASM International, Vol. 13, $1107-1112$.

International Electrotechnical Commission, 68-2 42, Test $\mathrm{K}_{\mathrm{c}} \mathrm{SO}_{2}$

International Electrotechnical Commission, 68-2-43, Test $\mathrm{K}_{d} \mathrm{H}_{2} \mathrm{~S}$

JEIDA-32, Japanese Electronic Industry Development Association. (1974). Standard on $\mathrm{H}_{2} \mathrm{~S}$, Japón.

JEIDA-25, Japanese Electronic Industry Development Association. (1974). Standard on $\mathrm{SO}_{2}$, Japón

Lázaro-López C. (1996). Determinación de la corrosión en el interior de los edificios de Ciudad Universitaria. Tesis Facultad de Química, Universidad Nacional Autónoma de México.

Oesh S. y Faller M. (1997). Environmental Effects on Materials. Corrosion Sci., Vol. 39, 105.

Rice D.W., Cappell R.J., Kinsolving W. y Laskowski J.J. (1980). Indoor Corrosion of Metals. J. Electrochemical Soc., Vol.127,891.

Rodríguez L.M., Genesca Ll.J. y Uruchurtu Ch.J. (1999). Corrosividad Atmosférica. Plaza y Valdez Editores- UNAM.

White E., Slensky G. y Dobbs B. (1987). Case Histories and Failures of Electronics and Communications Equiplment. Metals Handbook, ASM International, Vol. 13, 1113-1126.
Zakipour S. y Leygraf C. (1986). Evaluation of Laboratory Tests to Simulate Indoor Corrosion of Electrical Contac Materials. Electrochemical Society, Vol 133, 21.

Zakipour S., Tidblad J. y Leygraf C. (1997). Atmospheric Corrosion Effects of $\mathrm{SO}_{2}, \mathrm{NO}_{2}$, and $\mathrm{O}_{3}$. Electrochemical Society, Vol. 144, 3513.

\section{Bibliografía sugerida}

Abbott (1989). Corrosion of Electrical Contacts: Review of Flowing Mixed Gas Test Developments. British Corrosion J., Vol. 14, 153

Arroyave C. y Morcillo M. (1995). The Effect of Nitrogen Oxides in Atmospheric Corrosion of Metals. Corrosion Sci, Vol. 37, 293.

Genescá J. y Rodriguez C. (1992). Calibración de la agresividad del suroeste de la Ciudad de México., Revista Internacional de Contaminación Ambiental, Vol. 8, 81 .

Pourbaix M. y Pourbaix A.(1989). Recent Progress in Atmospheric Corrosion Testing. Corrosion Engineering, Vol. 45, 71 .

Sharma S.P., Thomas J.H. y Bader F.E. (1978). Development of a Gentle Accelerated Corrosion Test. J. Electrochemical Soc., Vol. 125, 2002.

Sinclair J.D. (1988). Corrosion of Electronics. J. Electrochemical Soc., Vol. 135, 89c.

Smith R.D. An Accelerated Atmospheric Corrosion Test (AACT), Atmospheric Corrosion of Enginnering Metals ASTM STP 646, 160.

Walter G.W. (1991). Laboratory Simulation of Atmospheric Corrosion by $\mathrm{SO}_{2}$ I. Corrosion Science, Vol. 32, 1331 
DOI: http://dx.doi.org/10.22201/fi.25940732e.2002.03n4.015

\section{Semblanza de los autores}

Armando Ortiz-Prado. Es ingeniero mecánico electricista y maestro en ingeniería mecánica por la Facultad de Ingeniería, UNAM. En 1989, obtuvo la distinción Universidad Nacional para jóvenes académicos en el área de docencia en ciencias exactas. Ha dirigido más de 70 tesis entre licenciatura y posgrado, así como más de 50 estudios y proyectos para el sector industrial en el área de manufactura, materiales y, en particular, análisis de falla. Tiene más de 40 publicaciones relacionadas con el área de su especialización en foros nacionales e internacionales. Actualmente es profesor y coordinador de la Unidad de Investigación y Asistencia Técnica en Materiales.

Rafael Schouwenaars. Maestro en ingeniería de minas por la Universidad de Lovaina, Bélgica. Durante cuatro años estuvo activo como investigador en varios proyectos relacionados con el estudio de la textura cristalográfica en aceros a bajo carbono en el Departamento de Metalurgia e Ingeniería de Materiales de la misma universidad. Llegó a México como experto en microscopía electrónica de barrido en el marco de una colaboración entre la Universidad de Gante y la UNAM. Desde hace dos años es profesor en la UNAM y se dedica al estudio de problemas metalúrgicos en la industria metal-mecánica mexicana, especializándose en análisis de falla y el comportamiento mecánico de materiales.

Sara Mercedes Cerrud-Sánchez. Es ingeniera mecánica electricista (industrial) y maestra en ingeniería mecánica por la Facultad de Ingeniería de la UNAM. Ha participado en más de 25 estudios, proyectos y asesorías para el sector industrial. Fungió como corresponsable en el proyecto Sistema experto para el análisis de falla de piezas mecánicas, metálicas. Tiene más de 25 publicaciones y actualmente es profesora del área de manufactura y materiales. 